\title{
Glucuronidation by UGT1A1 Is the Dominant Pathway of the Metabolic Disposition of Belinostat in Liver Cancer Patients
}

\author{
Ling-Zhi Wang ${ }^{1,2}$, Jacqueline Ramírez ${ }^{3}$, Winnie Yeo ${ }^{4}$, Mei-Yi Michelle Chan ${ }^{5}$, Win-Lwin Thuya ${ }^{1}$, Jie-Ying \\ Amelia Lau ${ }^{1}$, Seow-Ching Wan ${ }^{1}$, Andrea Li-Ann Wong ${ }^{6}$, Ying-Kiat Zee ${ }^{6}$, Robert Lim ${ }^{6}$, Soo-Chin Lee ${ }^{1,6}$, \\ Paul C. Ho ${ }^{5}$, How-Sung Lee ${ }^{2}$, Anthony Chan ${ }^{4}$, Sherry Ansher ${ }^{7}$, Mark J. Ratain ${ }^{3,8,9}$, Boon-Cher Goh ${ }^{1,2,6 *}$ \\ 1 Cancer Science Institute of Singapore, National University of Singapore, Singapore, Singapore, 2 Department of Pharmacology, National University of Singapore, \\ Singapore, Singapore, 3 Department of Medicine, The University of Chicago, Chicago, Illinois, United States of America, 4 Department of Clinical Oncology, Chinese \\ University of Hong Kong, Hong Kong, China, $\mathbf{5}$ Department of Pharmacy, National University of Singapore, Singapore, Singapore, $\mathbf{6}$ Department of Hematology \& \\ Oncology, National University Health System, Singapore, Singapore, $\mathbf{7}$ Cancer Therapy Evaluation Program, Bethesda, Maryland, United States of America, 8 Committee on \\ Clinical Pharmacology and Pharmacogenomics, The University of Chicago, Chicago, Illinois, United States of America, $\mathbf{9}$ Comprehensive Cancer Center, The University of \\ Chicago, Chicago, Illinois, United States of America
}

\begin{abstract}
Belinostat is a hydroxamate class HDAC inhibitor that has demonstrated activity in peripheral T-cell lymphoma and is undergoing clinical trials for non-hematologic malignancies. We studied the pharmacokinetics of belinostat in hepatocellular carcinoma patients to determine the main pathway of metabolism of belinostat. The pharmacokinetics of belinostat in liver cancer patients were characterized by rapid plasma clearance of belinostat with extensive metabolism with more than 4-fold greater relative systemic exposure of major metabolite, belinostat glucuronide than that of belinostat. There was significant interindividual variability of belinostat glucuronidation. The major pathway of metabolism involves UGT1A1-mediated glucuronidation and a good correlation has been identified between belinostat glucuronide formation and glucuronidation of known UGT1A1 substrates. In addition, liver microsomes harboring UGT1A ${ }^{*} 28$ alleles have lower glucuronidation activity for belinostat compared to those with wildtype UGT1A1. The main metabolic pathway of belinostat is through glucuronidation mediated primarily by UGT1A1, a highly polymorphic enzyme. The clinical significance of this finding remains to be determined.Trial Registration: ClinicalTrials.gov NCT00321594 http://clinicaltrials. gov/ct2/show/NCT00321594
\end{abstract}

Citation: Wang L-Z, Ramírez J, Yeo W, Chan M-YM, Thuya W-L, et al. (2013) Glucuronidation by UGT1A1 Is the Dominant Pathway of the Metabolic Disposition of Belinostat in Liver Cancer Patients. PLoS ONE 8(1): e54522. doi:10.1371/journal.pone.0054522

Editor: John Luk, Johnson \& Johnson Medical, China

Received August 2, 2012; Accepted December 12, 2012; Published January 30, 2013

Copyright: (c) 2013 Wang et al. This is an open-access article distributed under the terms of the Creative Commons Attribution License, which permits unrestricted use, distribution, and reproduction in any medium, provided the original author and source are credited.

Funding: The study was sponsored by the National Research Foundation of Singapore (Experimental Therapeutics Program) and the National Medical Research Council of Singapore (NMRC/CSA/021/2010). The funders had no role in study design, data collection and analysis, decision to publish, or preparation of the manuscript.

Competing Interests: The authors have declared that no competing interests exist. Please note that belinostat is a Topotarget product. This does not alter the authors' adherence to all the PLOS ONE policies on sharing data and materials.

*E-mail: phcgbc@nus.edu.sg

\section{Introduction}

Histone deacetylase inhibitors (HDACis) have been shown to have anticancer activity in malignant cells through acetylation of both histone and non-histone proteins. Acetylation of histones mediates epigenetic modulation of genes that can induce apoptosis, inhibit cell growth and reduce neoangiogenesis [1-7]. Several HDACis have been approved for treatment of peripheral or cutaneous T-cell lymphoma [8-10].

Belinostat is a hydroxamic acid based pan-histone deacetylase inhibitor in clinical development as an anti-cancer therapy in a range of hematological and solid malignancies, both as single agent as well as combination therapies [11-18]. Currently belinostat has been granted fast track and orphan drug designation by the U.S. Food and Drug Administration for treatment of relapsed or refractory peripheral T-cell lymphoma. Although clinically the drug has been relatively well tolerated, the common adverse events included fatigue, nausea and vomiting, and lethargy, reaching grade 3 at the maximum tolerable dose of $1200 \mathrm{mg} / \mathrm{m}^{2}$ given in 30 minute ( $\mathrm{min}$ ) infusions for 5 consecutive days every 3 weeks. Belinostat is available in both oral and intravenous formulations, and as the drug is expected to be administered on a chronic basis, cumulative toxicities are possible. Therefore, it would be important to determine the metabolism of belinostat, to understand its in vivo disposition. Up till now, very little is known about the metabolic pathways of belinostat.

Other members of the hydroxamic acid class HDAC inhibitors like vorinostat and panobinostat undergo primary metabolism by glucuronidation [19-21]. On the other hand, romidepsin, a cyclic tetrapeptide class HDAC inhibitor, undergoes metabolism mainly by CYP3A4 [22]. Therefore, we hypothesized that glucuronidation would be the primary pathway for metabolism of belinostat. Furthermore, many glucuronosyl transferase isoforms are highly polymorphic and impact function, for example, homozygous deletion of UGT2B17 alleles (UGT2B17*2) results in defective 
metabolism of vorinostat [23]. Accordingly, it would be important to identify the main drug metabolizing isoform of belinostat, to better understand the influence of pharmacogenetics on the interindividual variability of belinostat pharmacodynamics. This also provides guidance for further drug interaction studies.

In this study, we studied the pharmacokinetics of belinostat and identified its metabolites based on the mass spectra and maximum UV absorption. We identified the dominant metabolite of belinostat as belinostat glucuronide, and further studied the glucuronidation of belinostat using a panel of UGT isoenzymes and human liver microsomes, to determine the main isoform responsible for its metabolism. Finally, potential pharmacogenetic influence on pharmacodynamics of belinostat was explored.

\section{Materials and Methods}

The protocol for this trial is available as supporting information; see Protocol S1.

\section{Reagents}

Belinostat (PXD101) was a gift from the National Cancer Institute (Bethesda, MD, USA). Belinostat glucuronide (belinostatG) was chromatographically separated with HPLC-UV and isolated from human plasma using a fraction collector. Its chemical structure and purity has been verified through LCMS/MS (API 4000 triple-quadrupole mass spectrometer; AB Sciex, Concord, Canada) and HPLC-UV analysis. Vorinostat glucuronide (vorinostat-G), the internal standard, was a gift from Merck Sharp \& Dohme (I.A.) Corp. Acetonitrile (HPLC grade), methanol (HPLC grade), ethanol (analytical grade), formic acid (analytical grade), di-sodium hydrogen phosphate dihydrate $\left(\mathrm{Na}_{2} \mathrm{HPO}_{4} 2 \mathrm{H}_{2} \mathrm{O}\right)$ and orthophosphoric acid $(85 \%)$ were obtained from Merck (Darmstadt, Germany). Direct-QTM water (Millipore Milford, MA, USA) was used for the mobile phase preparation. Human UGT supersomes and UGT Reaction-Solutions A and B were purchased from BD Gentest (San Jose, CA, USA). These are cDNA expressed UGTs and a panel of 12 (UGT1A1, UGT1A3, UGT1A4, UGT1A6, UGT1A7, UGT1A8, UGT1A9, UGT1A10, UGT2B4, UGT2B7, UGT2B15, UGT2B17) was used.

\section{Human Liver Microsome Preparation}

Human livers from 37 normal Caucasian donors were processed in Dr. Mary Relling's laboratory at St. Jude Children's Research Hospital (Memphis, TN, USA) and were provided by the Liver Tissue Procurement and Distribution System (funded by \#N01DK-9-2310) and by the Cooperative Human Tissue Network. The 37 human liver microsomes (HLM) were isolated from different liver specimen with no correlation each other. Microsomes were prepared by differential centrifugation.

\section{Study Cohorts}

This multicentre phase I trial of belinostat was done in Hong Kong and Singapore. All patients provided written informed consent according to Good Clinical Practice guidelines, and the protocol was approved by the institutional review boards of Prince of Wales Hospital, Hong Kong and National University Hospital, Singapore. Seventeen patients were treated with belinostat at escalating doses of $600(n=3), 900(n=3), 1,200(n=6), 1,400$ $(\mathrm{n}=5) \mathrm{mg} / \mathrm{m}^{2}$ daily by intravenous (i.v.) infusion over $30 \mathrm{~min}$ for 5 days every 21 days.

\section{Pharmacokinetic Assessment}

Blood specimens were collected at predetermined sampling time points on day 1 at predose, $15 \mathrm{~min}, 30 \mathrm{~min}, 45 \mathrm{~min}, 1$ hour (h),
$1.5 \mathrm{~h}, 2 \mathrm{~h}, 3 \mathrm{~h}, 5 \mathrm{~h}$ and $24 \mathrm{~h}$ postinfusion and day 5 at predose, $30 \mathrm{~min}, 1 \mathrm{~h}, 1.5 \mathrm{~h}, 3 \mathrm{~h}, 5 \mathrm{~h}$ postinfusion and day 22 following the start of 30 min i.v. infusion in a phase I clinical trial of belinostat. Venous blood samples had been collected in heparinized tubes. Collected blood samples were centrifuged at $3000 \mathrm{~g}$ for 10-15 min and the plasma (supernatant) was separated from the cell pellet and stored in plain tubes at $-80^{\circ} \mathrm{C}$ till analysis. The concentrations of belinostat and belinostat glucuronide were quantified by a modified high-performance liquid chromatography/mass spectrometry method [24]. Briefly, vorinostat-G was used as the internal standard for belinostat-G. The mass spectrometer was operated in positive ion mode with the optimal mass transitions of belinostat-G: $\mathrm{m} / z \quad 495>93$ and vorinostat-G: $\mathrm{m} / z \quad 441>232$, respectively. The analytical method was well validated with good linearity (coefficient of determination, $r^{2} \geq 0.999$ ) in the range of $1-$ $100 \mu \mathrm{M}$ for belinostat-G.

\section{Identification of Belinostat Metabolites and Isolation of Belinostat-G}

Metabolite screening in patient samples was processed with HPLC-UV at $268 \mathrm{~nm}$, the maximum absorption wavelength of belinostat. Baseline separation of all the analytes was achieved on the Alltima $\mathrm{C}_{18}(150 \mathrm{~mm} \times 2.1 \mathrm{~mm}, 5 \mu \mathrm{m})$ column. Mobile phase solvent A was $20 \mathrm{mM} \mathrm{Na}_{2} \mathrm{HPO}_{4}(\mathrm{pH} 4.2$, adjusted by orthophosphoric acid), while mobile phase solvent $\mathrm{B}$ comprised of $70 \%$ acetonitrile and $30 \%$ methanol $(\mathrm{v} / \mathrm{v})$. A gradient mobile phase programme was used - initial percentage of solvent A was $75 \%$ (v/ v) and that of solvent B was 25\% (v/v); percentage of solvent B was increased to $95 \%(\mathrm{v} / \mathrm{v})$ over $13 \mathrm{~min}$ and immediately switched back to $25 \%(\mathrm{v} / \mathrm{v})$ for $7 \mathrm{~min}$ before injection of the subsequent sample. Total run time of each sample was $25 \mathrm{~min}$. The flow rate was maintained at $0.5 \mathrm{~mL} / \mathrm{min}$.

The potential belinostat metabolites were further verified with a tandem mass spectrometer in Q1, product and precursor scans. The isolation and purification of the major metabolite of belinostat was performed on a Nova-Pak $3.9 \times 300 \mathrm{~mm}$ C18 column (Waters, Ireland), using $20 \mathrm{mM}$ ammonium acetate buffer $(\mathrm{pH} 5.0)$ and $100 \%$ methanol in an initial mobile phase composition of $60 \%: 40 \%(\mathrm{v} / \mathrm{v})$ with a flow rate of $0.6 \mathrm{~mL} / \mathrm{min}$. Methanol was increased to $95 \%$ over $10 \mathrm{~min}$ and immediately switched back to $40 \%$ for $6 \mathrm{~min}$ before the next injection. $\beta$-Glucuronidase hydrolysis and mass spectrometry (both in positive and negative mode) to ascertain purity and identity of the major metabolite.

\section{Acidic Stability Test for Belinostat-G}

$1 \mathrm{mg} / \mathrm{mL}$ of belinostat- $\mathrm{G}$ and vorinostat-G was prepared in various vehicles with different $\mathrm{pH}$ values. These vehicles included $10 \mathrm{mM}$ ammonium acetate $(\mathrm{pH}=6.5), 10 \mathrm{mM}$ ammonium formate $(\mathrm{pH}=6.0), 0.1 \%$ acetic acid $(\mathrm{pH}=3.2)$ and $0.1 \%$ formic acid $(\mathrm{pH}=2.6)$. All of these test samples were incubated at $37^{\circ} \mathrm{C}$ for $24 \mathrm{~h}$. Milli $\mathrm{Q}$ water $(\mathrm{pH}=5.5)$ was used as control solution which was stored in $-20^{\circ} \mathrm{C}$. All the samples were analyzed by LCMS/MS to obtain the values of peak area for belinostat-G and vorinostat-G. The acidic stability of both conjugated compounds was evaluated through comparison of peak area ratio of test samples versus control.

\section{In Vitro Potency Assessment on Belinostat and Belinostat-G}

To determine the presence or absence of dose-dependent activity of belinostat and belinostat-G against hepatocellular cancer cells, human hepatocellular liver carcinoma (HepG2) cells were seeded in two 24-well plates at 10,000 cells per well. To one 
plate, 5 different concentrations of belinostat were added to obtain 2 replicates per concentration $(0.1,0.5,1,2,5,10 \mu \mathrm{M})$. To the other plate, equal molar concentrations of belinostat-G were added similarly. After 72 h, cells were stained with Gentian Violet paint $(0.5 \% \mathrm{w} / \mathrm{v}$; B.P.) and assessed for viability. Furthermore, the sensitivities of HepG2 to belinostat and belinostat-G were quantitatively measured using the MTS assay. In brief, HepG2 cells seeded in 96-well plates at 3,000 cells/well were treated with seven different doses of belinostat or belinostat-G and then assessed for growth inhibition with Promega CellTiter $96^{\circledR}$ AQueous Non-Radioactive Cell Proliferation Assay. After incubating $24 \mathrm{~h}, 100-\mu \mathrm{L}$ drug solution or blank medium was added into each well of 96-well plates. $72 \mathrm{~h}$ later, $20 \mu \mathrm{L}$ of MTS/PES was added into each well for incubation for $3 \mathrm{~h}$. OD values are measured on wavelength $490 \mathrm{~nm}$. Cell growth inhibition was calculated based on the luminescent units with subtraction of background reading for only medium containing wells. The growth inhibition was defined as the percentage of the average luminescent units from drug-treated wells over that from control wells without treatment. The experiment was done in triplicates. $\mathrm{IC}_{50}$ values were calculated using GraphPad PRISM software (GraphPad Software, Inc., La Jolla, CA, USA).

\section{Western Blotting}

For western blot analysis, the HepG2 cells treated with belinostat were harvested and lysed in cell lysis buffer containing $50 \mathrm{mM}$ Tris HCl, pH 7.4, $150 \mathrm{mM} \mathrm{NaCl}, 1 \mathrm{mM}$ EDTA, $1 \%$ TRITON X-100 (Sigma-Aldrich) and protease inhibitor cocktail (Roche). Protein concentrations were quantified using the Bradford assay (Invitrogen). Proteins were separated on a $12 \%$ SDS-PAGE gel and transferred to nitrocellulose membrane. Nonspecific protein binding was blocked by incubating with $5 \%$ non-fat milk (BioRad Laboratories) in $0.1 \%$ PBS-Tween (PBST) at room temperature for $1 \mathrm{~h}$ and incubated with specific antibodies, Histone H3 and Ac-H3 [K9/K14] (Cell Signaling), at $4^{\circ} \mathrm{C}$ overnight. Appropriate species-specific Horseradish peroxidisesconjugated secondary antibodies were then added and incubated at room temperature for $1 \mathrm{~h}$ followed by detection using an enhanced chemiluminescence kit (GE Healthcare).

\section{Screening of 12 Human UGT Supersomes and Kinetic Analysis}

A typical incubation consisted of $50 \mu \mathrm{g}$ of UGT, $2 \mathrm{mM}$ UDPGA (UGT Reaction Solution A), $50 \mathrm{mM}$ Tris-HCl, $8 \mathrm{mM}$ $\mathrm{MgCl}_{2}$ and $0.025 \mathrm{mg} / \mathrm{ml}$ alamethicin (UGT Reaction Solution B) in a final incubation volume of $100 \mu \mathrm{L}$. Before the addition of substrate, the incubation mixture was prewarmed and equilibrated for $5 \mathrm{~min}$ at $37^{\circ} \mathrm{C}$. Reactions were initiated by addition of $10 \mu \mathrm{M}$ belinostat solution in DMSO and incubated at $37^{\circ} \mathrm{C}$ for $30 \mathrm{~min}$; the reactions were terminated by adding $200 \mu \mathrm{L}$ of ice-cold methanol and vortexing. The incubation mixture was vortexed and centrifuged $(10,060 \mathrm{~g})$ at $4^{\circ} \mathrm{C}$ for $10 \mathrm{~min}$, and the supernatants were analyzed. Insect microsomes without UGT cDNA served as negative control. Kinetic parameters $\left(K_{\mathrm{m}}\right.$ and $\left.V_{\max }\right)$ of belinostat glucuronidation by UGT1Al supersomes were determined using substrate concentrations of 10 to $750 \mu \mathrm{M}$. Incubations were performed as described above. Three independent experiments were performed for kinetic analysis.

\section{Correlation Study with UGT1A1 Substrates, UGT1A1 Gene Expression and UGT1A1*28 Polymorphism}

In a correlation study of glucuronidation of belinostat and UGT1A1 substrates, belinostat glucuronidation was measured as described above using $100 \mu \mathrm{M}$ belinostat and $50 \mu \mathrm{g}$ HLM; this concentration of belinostat was selected as it is close to the $\mathrm{Km}$ of UGT1A1. Glucuronidation of bilirubin, thyroxine and SN-38 in these microsomes, measurement of UGT1A1 gene expression and genotyping for UGT1A1*28 have been previously described [2527].

\section{Data Analysis}

Belinostat-G formation velocity (v) was calculated as $\mathrm{Cm}$, $30 \mathrm{~min} /$ incubation time/CYP concentration, where $\mathrm{Cm}, 30 \mathrm{~min}$ was the metabolite concentration after $30 \mathrm{~min}$ incubation. Plots of substrate concentration, $\mathrm{S}(\mathrm{X}$ axis) versus $v$ ( $\mathrm{Y}$ axis) were then constructed. $\mathrm{Km}$ and Vmax were calculated using the MichaelisMenten equation (GraphPad software, Inc., San Diego, CA, USA).

$$
v=[\mathrm{S}] \times \mathrm{Vmax} /([\mathrm{S}]+\mathrm{Km})
$$

Association between belinostat glucuronide concentration and UGT1Al substrates were performed using both Pearson's correlation and Spearman's correlation tests (GraphPad Prism software, La Jolla, CA). Pharmacokinetic parameters were estimated based on non-compartmental analysis model using WinNonlin software version 5.3 (Pharmsight, Sunnyvale, CA, USA). Relative exposure of belinostat-G in patient plasma was represented by the molar concentration AUC ratio of belinostat-G over belinostat. One way analysis of variance (ANOVA) was used to determine the significance among the genotypes with Tukey posthoc tests for adjustment for comparison between individual groups. A value of $\mathrm{p}<0.05$ was considered to be significant.

\section{Results}

\section{Pharmacokinetics and Metabolism of Belinostat in Human Plasma}

The concentrations of belinostat and belinostat-G in 17 patients enrolled in a phase I clinical trial in hepatocellular carcinoma (HCC) were quantified for estimation of pharmacokinetic parameters (Table 1). Belinostat followed linear pharmacokinetics at doses ranging from 600 to $1400 \mathrm{mg} / \mathrm{m}^{2}$, with larger interindividual variability of clearance at higher doses. The coefficients of variation of Clearance (CL) were $29.0 \%$ and $30.6 \%$ at 1200 and $1400 \mathrm{mg} / \mathrm{m}^{2}$, respectively. Belinostat was eliminated rapidly after i.v. administration due to intensive phase II glucuronide conjugation, M1 (Figure 1).

Five metabolites of belinostat were identified through comparing the ultraviolet (UV) absorption of plasma samples at $268 \mathrm{~nm}$ before and after dosing. Similar chromatogram profiles were detected on day 1 (Figure 1B) and day 5 (Figure 1A). The MS/MS product ions of these five metabolites monitored in positive mode supported their chemical structure identification (Table 2). Glucuronidation was the most significant pathway of belinostat metabolism; two alternate biotransformation pathways involved methylation to methyl belinostat and reduction of the hydroxamic group to its corresponding belinostat amide. Belinostat acid and belinostat glucoside were 2 other minor metabolites detected. The proposed metabolic pathway of belinostat is indicated in Figure 2. The chemical structures of these metabolites are proposed based on their protonated molecule mass peaks $[\mathrm{M}+\mathrm{H}]{ }^{+}$, 2-fold molecule mass peaks $[2 \mathrm{M}+\mathrm{H}]{ }^{+}$and mass fragmentation profiling generated by product scan. These structures were further confirmed by analysis of fragmentation ions of precursor ions, which revealed a common product ion $(\mathrm{m} / \mathrm{z} 93$, phenylamino 


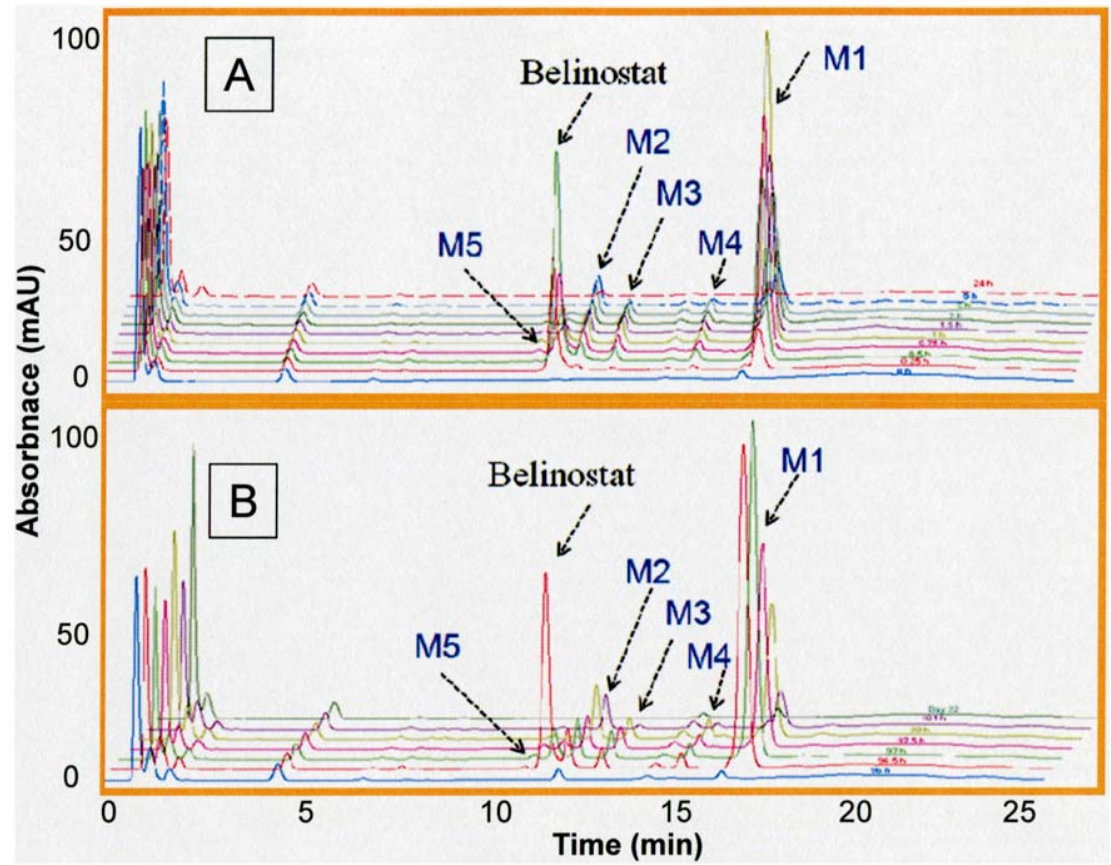

Figure 1. Identification of belinostat metabolites in human plasma using HPLC-UV at maximum absorption wavelength $(\lambda=\mathbf{2 6 8} \mathbf{~ n m})$. Chromatogram at day 5 and day $22(A)$; day1(B). doi:10.1371/journal.pone.0054522.g001

fragment) of belinostat and its five metabolites. Based on the metabolite structures, the hydroxamide moiety is the key structure for the potency of belinostat. Scanning of $\mathrm{m} / \mathrm{z}$ spectra from the plasma of patients supported glucuronidation of belinostat through detection of 495, 989, 493 representing the protonated belinostat $\mathrm{G}$, protonated double belinostat $\mathrm{G}$, and deprotonated belinostat $\mathrm{G}$, respectively. The chemical structure of belinostat-G isolated from plasma was elucidated by mass spectrometry. A series of mass spectra of belinostat-G were acquired to propose the chemical structure. For instance, m/z 495 and m/z 989 were identified as its protonated molecule mass peaks $[\mathrm{M}+\mathrm{H}]^{+}$and 2-fold molecule mass peaks $[2 \mathrm{M}+\mathrm{H}]^{+}$, respectively. Scanning in negative mode detected its corresponding deprotonated parent ion as $\mathrm{m} / \mathrm{z}$ $493[\mathrm{M}-\mathrm{H}]^{-}$. A similar mass fragmentation profiling in positive mode was found both for belinostat and belinostat-G. Based on the common product ion (phenylamino fragment), a precursor ion scan of $\mathrm{m} / \mathrm{z} 93$ was processed on belinostat and belinostat-G to derive their parent ions as $\mathrm{m} / \mathrm{z} 319[\mathrm{M}+\mathrm{H}]^{+}$for belinostat and $\mathrm{m} /$ z $495[\mathrm{M}+\mathrm{H}]^{+}$for belinostat-G, respectively. Assuming this was correct, the chemical structures of its metabolites would be expected to possess similar UV spectra for these metabolites and its parent compound as well due to similarity on their basic molecular structures, and this was supported by UV spectral analysis generated by diode array detector (DAD) showing similar maximum wavelength at $268 \mathrm{~nm}$ for belinostat and its five metabolites.

The major metabolite was identified as belinostat-G which was further confirmed through enzyme hydrolysis reaction. The

Table 1. Pharmacokinetic parameters for belinostat after 30 min i.v. infusion in Phase I trial.

\begin{tabular}{|c|c|c|c|c|c|}
\hline Substance & Parameter* & $600 \mathrm{mg} / \mathrm{m}^{2}$ & $900 \mathrm{mg} / \mathrm{m}^{2}$ & $1200 \mathrm{mg} / \mathrm{m}^{2}$ & $1400 \mathrm{mg} / \mathrm{m}^{2}$ \\
\hline \multirow[t]{7}{*}{ Belinostat } & No. of Patients & 3 & 3 & 6 & 5 \\
\hline & $\mathrm{Cmax}, \mu \mathrm{mol} / \mathrm{L}$ & $88.2 \pm 8.5$ & $98.5 \pm 29.7$ & $153.5 \pm 50.4$ & $174.4 \pm 48.3$ \\
\hline & Tmax, h & $0.33 \pm 0.14$ & $0.42 \pm 0.14$ & $0.64 \pm 0.10$ & $0.40 \pm 0.14$ \\
\hline & $\mathrm{AUC}_{0-24 \mathrm{~h}}, \mathrm{~h} \times \mu \mathrm{mol} / \mathrm{L}$ & $61.3 \pm 1.2$ & $70.0 \pm 17.3$ & $112.6 \pm 37.1$ & $149.0 \pm 46.2$ \\
\hline & $\mathrm{T}_{1 / 2}, \mathrm{~h}$ & $3.54 \pm 0.34$ & $4.07 \pm 0.39$ & $4.14 \pm 0.42$ & $3.54 \pm 0.50$ \\
\hline & $\mathrm{CL}, \mathrm{L} / \mathrm{h}$ & $52.6 \pm 3.8$ & $70.5 \pm 17.9$ & $54.6 \pm 15.8$ & $53.0 \pm 16.2$ \\
\hline & $\mathrm{Vz}, \mathrm{L}$ & $268.3 \pm 26.6$ & $409.2 \pm 76.7$ & $304.9 \pm 185.6$ & $279.1 \pm 120.8$ \\
\hline Belinostat-G & $\mathrm{AUC}_{0-24 \mathrm{~h}}, \mathrm{~h} \times \mu \mathrm{mol} / \mathrm{L}$ & $286.5 \pm 36.6$ & $302.1 \pm 54.8$ & $514.1 \pm 41.4$ & $575.1 \pm 154.0$ \\
\hline Relative Exposure & Bel-G/Bel AUC ratio & $4.79 \pm 0.46$ & $4.22 \pm 0.15$ & $4.82 \pm 1.80$ & $4.13 \pm 0.68$ \\
\hline
\end{tabular}

Abbreviation: Cmax, maximum concentration; Tmax, time to maximum concentration; $A \cup C_{0-24}$, area under the curve from 0 to $24 \mathrm{~h}$; $\mathrm{T}_{1 / 2}$, half-life at the elimination phase; $\mathrm{CL}$, clearance; $\mathrm{Vz}$, volume of distribution. ${ }^{*}$ Mean $\pm \mathrm{SD}$; AUC ratio was calculated based on $\mathrm{AUC}_{0-24 \mathrm{~h}}$ of Bel-G (belinostat-G) over $\mathrm{AUC} \mathrm{C}_{0-24 \mathrm{~h}}$ of Bel (belinostat). doi:10.1371/journal.pone.0054522.t001 


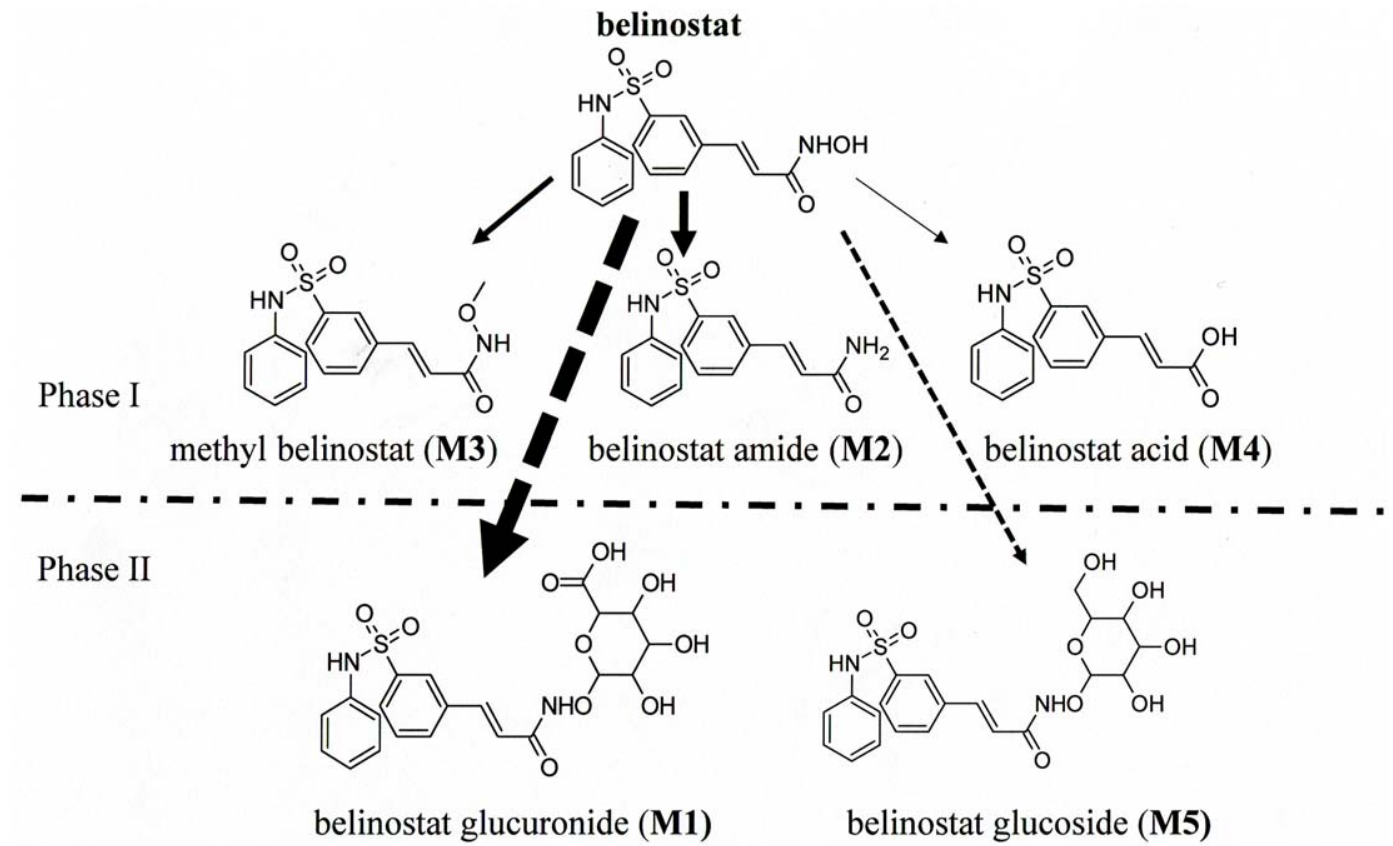

Figure 2. Belinostat metabolism pathway in human plasma with glucuronidation of belinostat as the dominant metabolism. doi:10.1371/journal.pone.0054522.g002

isolated belinostat-G from patient plasma samples was subjected to Escherichia coli-derived $\beta$-glucuronidase $(6.8 \mathrm{mg} / \mathrm{mL})$ incubation for $5 \mathrm{~h}$, and the resulting reaction mixture was analyzed through LC-MS/MS. The quantitative pharmacokinetic analysis indicated that belinostat-G exposure was 4-fold higher than that of belinostat in human plasma based on the molar concentration AUC ratio of belinostat-G/belinostat (Table 1). Hence, belinostat glucuronidation was confirmed to be the dominant metabolic pathway for belinostat.

\section{Stability of Belinostat-G in Acidic Environment}

To further characterize the glucuronidation of belinostat, we tested the acidic stability of belinostat-G. Belinostat-G and vorinostat-G, a parallel control for belinostat- $G$, were demonstrated to be stable after $24 \mathrm{~h}$ incubation at $37^{\circ} \mathrm{C}$ in various acidic solutions $(\mathrm{pH} 2.6-\mathrm{pH}$ 6.5). The variation in concentrations measured at the beginning of the experiment and after $24 \mathrm{~h}$ was very marginal $(<4 \%)$. This is consistent with our proposed $\mathrm{O}$ conjugated structure of belinostat-G (M1) shown in Figure 2. However, minor degradation $(7 \%)$ was identified only when the $\mathrm{pH}$ value was decreased to 2.6. The results confirmed belinostat is conjugated to glucuronide at the $\mathrm{O}$ position, as $\mathrm{O}$-glucuronides but not $\mathrm{N}$-glucuronides of hydroxamic acids are stable in acidic environment $[28,29]$. As vorinostat-G has been confirmed to be an O-conjugated glucuronide [30], it demonstrates that the isolated belinostat-G is an O-conjugated metabolite as well.

\section{Determination of In Vitro Cytotoxicity Effects of Belinostat and Belinostat-G}

Figure $3 \mathrm{a}$ and $3 \mathrm{~b}$ show the results of dose-increasing concentrations of belinostat and belinostat-G conducted on separate 24well plates after incubation for $72 \mathrm{~h}$. As belinostat concentration increased, number of viable cells (stained violet) showed dosedependent decrease. Conversely, increasing concentrations of belinostat-G did not seem to affect cell viability within the concentration range tested. As such, at equimolar concentrations between $0-10 \mu \mathrm{M}$, belinostat demonstrates significant dosedependent cytotoxicity while belinostat-G does not. Furthermore, the results of MTS assays were also consistent with that of the staining assay (Figure 3c). Histone H3 acetylation for belinostat exposure demonstrated a time and concentration dependent increase (Figure 3d).

Table 2. Identification of belinostat metabolites in human plasma using HPLC-DAD \& LC-MS/MS analyses.

\begin{tabular}{llllll}
\hline Peak ID & Retention Time $(\mathbf{m i n})$ & $\lambda(\mathbf{n m})$ & [M+H] ${ }^{+}$ & \multicolumn{1}{l}{ MS/MS } & Proposed Structure \\
\hline Belinostat & 11.5 & 268 & 319 & $93,204,268,286$ & Parent compound \\
M1 & 16.8 & 268 & 495 & $93,204,268,286,319$ & Belinostat glucuronide \\
M2 & 12.1 & 268 & 303 & $93,204,268,286$ & Belinostat amide \\
M3 & 13.0 & 268 & 333 & $93,204,268,286$ & Methyl belinostat \\
M4 & 15.1 & 268 & 304 & $93,268,286,319$ & Belinostat acid \\
M5 & 10.9 & 268 & 481 & $93,204,268,286,319$ &
\end{tabular}




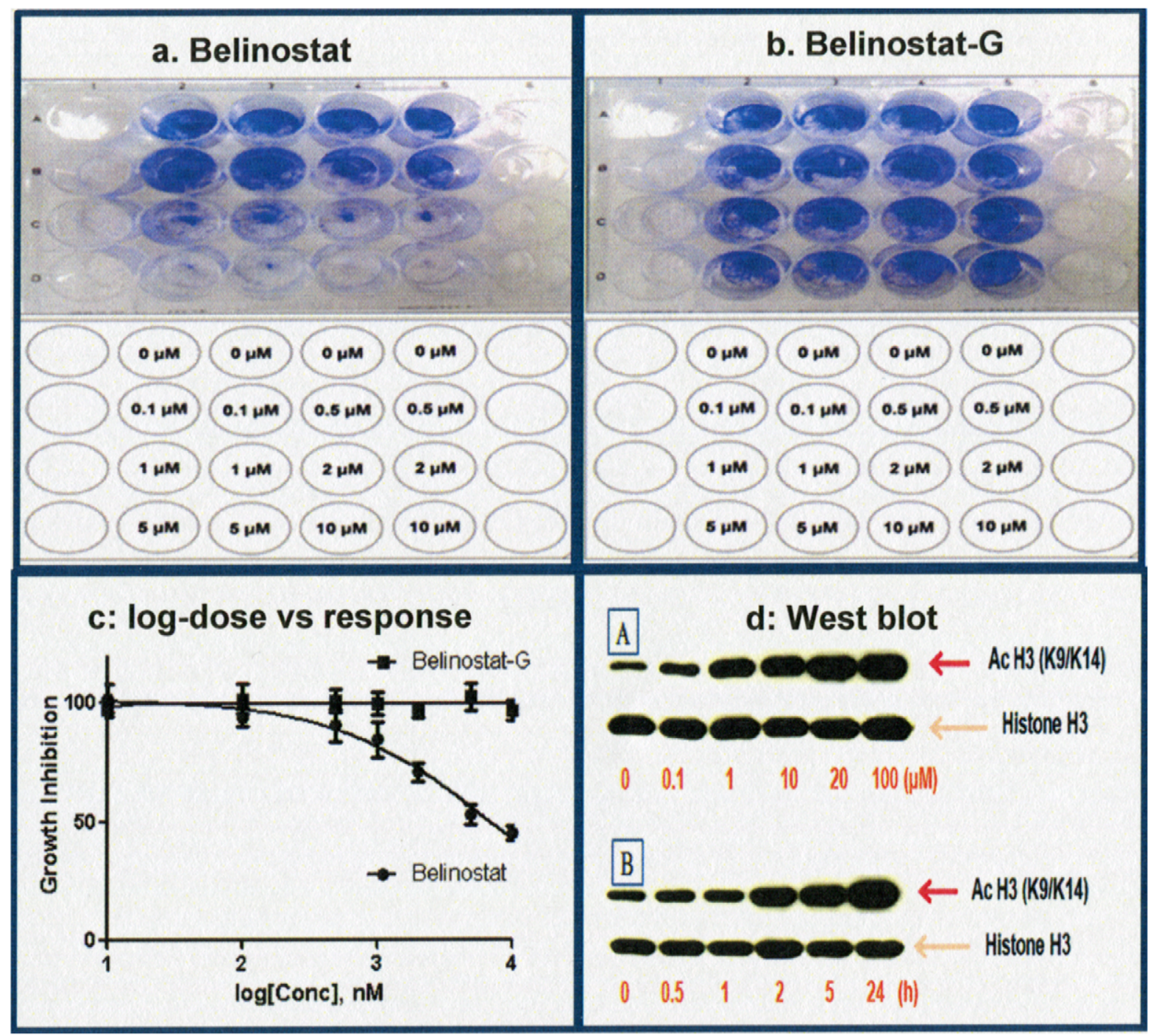

Figure 3. Cytotoxicity and acetylation activity on HepG2. a: belinostat incubation; b: belinostat-G incubation; (templates for concentrations added (lower) and results of 24-well dose-increasing concentrations on HepG2 Cells (upper). c: MTS results for belinostat (IC $50=6.4 \mu \mathrm{M})$ and belinostat-G (cannot be converged). d:Belinostat acetylation activity on HepG2 cells (western blot). A: Acetyl histone 3 increased with dose increment after $5 \mathrm{~h}$ incubation; B: Kinetic changes of acetyl histone 3 with time increment at $10 \mu \mathrm{M}$.

doi:10.1371/journal.pone.0054522.g003

\section{In Vitro Metabolism Screening of UGTs for Belinostat-G} Formation and Enzyme Kinetics

UGT1Al glucuronidated belinostat significantly (Figure 4A), whereas no metabolism was observed after incubation with UGT1A4, UGT1A6, UGT1A7, UGT1A9, UGT1A10, UGT2B15 and UGT2B17. Minor metabolism (less than 3\%) was detected with UGT1A3, UGT1A8, UGT2B4 and UGT2B7. $73.6 \%$ and $89.4 \%$ of belinostat was converted to belinostat-G after $2 \mathrm{~h}$ and $4 \mathrm{~h}$ of incubation with UGT1Al, respectively (Figure 4B). Enzyme kinetic parameters for the glucuronidation of belinostat were estimated by fitting the pooled data from the UGT1Al incubations performed in triplicate to the Michaelis-Menten equation. The apparent $\mathrm{Km}$ and $\mathrm{Vmax}$ values for the glucuronide formation were $99.6 \mu \mathrm{M}$ and $353.1 \mathrm{pmol} / \mathrm{min} / \mathrm{mg}$ protein, respectively (Figure 5). The intrinsic clearance $(\mathrm{Vmax} / \mathrm{Km})$ for formation of belinostat-G was estimated to be $3.5 \mu \mathrm{L} / \mathrm{min} / \mathrm{mg}$ protein. In the control insect microsomes, no belinostat-G formation was observed.

\section{Correlation of Glucuronidation of Belinostat with UGT} Substrates

In human liver microsomes, the association between glucuronidation of belinostat and UGT substrates were tested using parametric (Person's correlation) and non-parametric (Spearman's correlation) tests. Belinostat-G formation correlated strongly with bilirubin glucuronide formation (Figure 6A) (Pearson $\mathrm{r}^{2}=0.53$; Spearman $\left.\mathrm{r}^{2}=0.61 \mathrm{p}<0.0001\right)$ and other well established substrates of UGT1A1 like thyroxine and SN38 (Pearson $r^{2}=0.68$; Spearman $r^{2}=0.81$ and Pearson $r^{2}=0.82$; Spearman $\mathrm{r}^{2}=0.62$, respectively, all $\mathrm{p}<0.001$ ) (Figure $6 \mathrm{~B}$, and $6 \mathrm{C}$ ). Belinostat-G concentrations after incubation with the HLM correlated well with UGT1Al mRNA expression (Pearson, $r^{2}=0.66, p<0.0001$, Figure 7A). Taken together, these suggest that UGT1Al is the predominant UGT isoform in belinostat glucuronidation. 


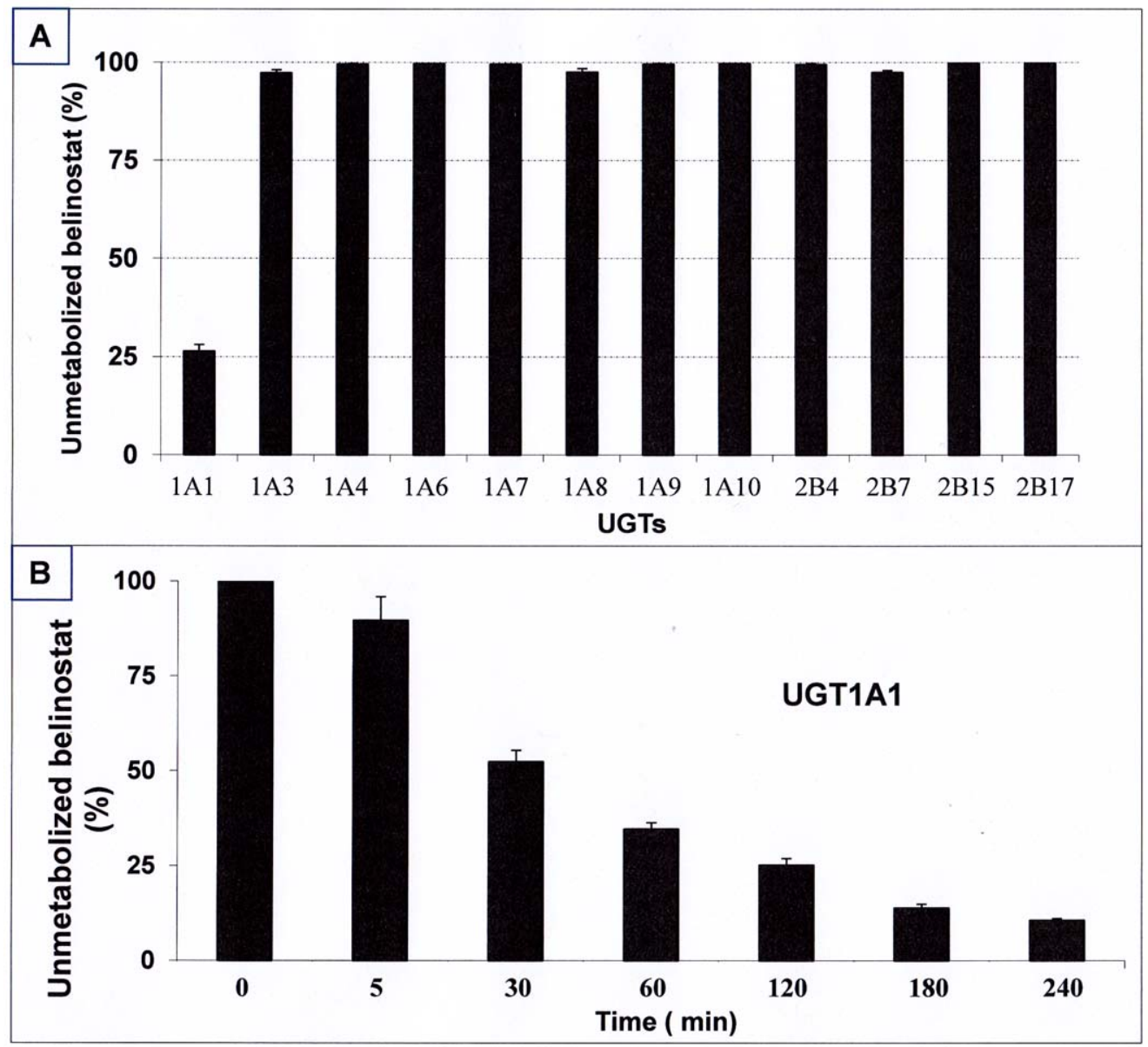

Figure 4. Enzyme stability test in a panel of 14 UGT isoforms after $2 \mathrm{~h}$ incubation at $37^{\circ} \mathrm{C}$ (A); Time course of glucuronidation of belinostat by UGT1A1supersomes at $37^{\circ} \mathrm{C}$ (B). doi:10.1371/journal.pone.0054522.g004

\section{Michaelis-Menten data}

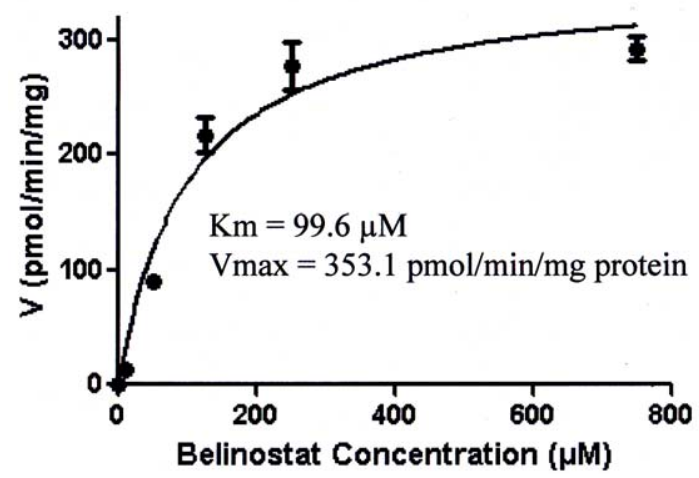

Figure 5. Enzyme kinetics of glucuronidation of belinostat by UGT1A1. The apparent $\mathrm{Km}$ and Vmax values for the glucuronide formation were $99.6 \mu \mathrm{M}$ and $353.1 \mathrm{pmol} / \mathrm{min} / \mathrm{mg}$ protein, respectively. doi:10.1371/journal.pone.0054522.g005
UGT1A1 Genotype and Glucuronidation of Belinostat in Human Liver Microsomes and Correlation with UGT1A1 Gene Expression

UGT1Al is a polymorphic enzyme with allelic variants that influence its enzymatic activity. UGT1A1*28 is a frequent polymorphism that reduces glucuronidation activity. Therefore, to determine the effect of UGT1A1*28 on formation of belinostat$\mathrm{G}$, we measured belinostat-G concentrations after incubation of belinostat with each of 37 HLM samples previously genotyped for $U G T 1 A 1 * 28$. The 30 -min post-incubation belinostat-G concentrations (mean $\pm \mathrm{SD}$ ) were 15.39 $\pm 6.00,11.35 \pm 4.11$ and $7.14 \pm 3.28 \mu \mathrm{mol}$ for UGT1A1*1 homozygous, UGT1A1*28 heterozygous and homozygous microsomes, respectively. One way ANOVA analysis showed that significant difference between groups was detected $(p=0.01)$. Tukey posthoc analysis suggested that only the difference between UGT1Al*1 and UGT1A $1 * 28$ homozygous microsomes was statistically significant. As shown in Figure 7B, UGT1A1*1 homozygous microsomes had 2 fold greater mean belinostat-G concentrations compared to UGT1A1*28 homozygous microsomes after incubation $(p<0.001)$.

\section{Discussion}

To our knowledge, this is the first report describing the glucuronidation of belinostat. We have demonstrated that belinostat undergoes extensive metabolism through glucuronida- 

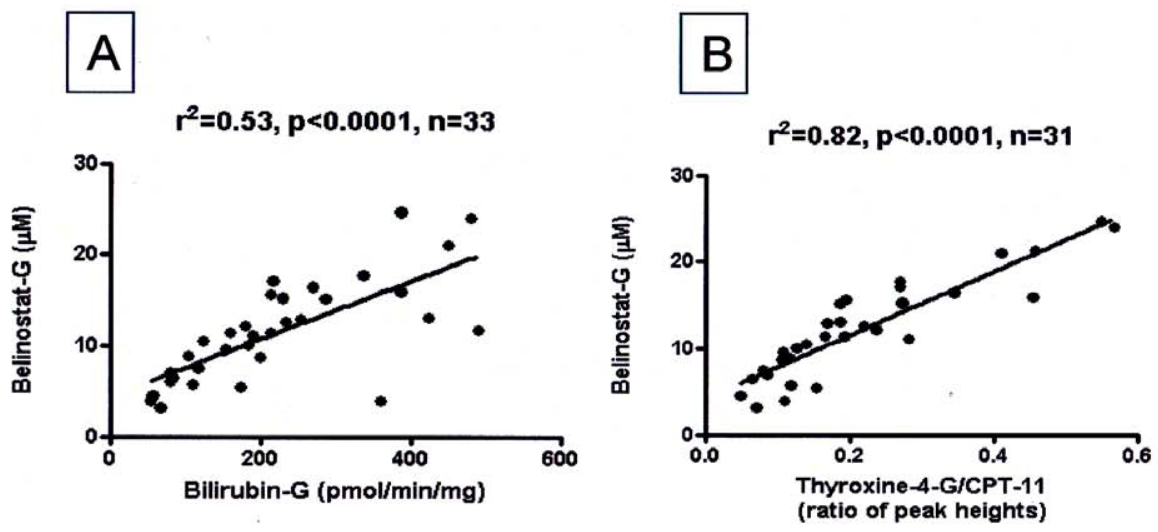

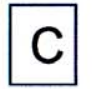

\section{$r^{2}=0.68, p<0.0001, n=37$}

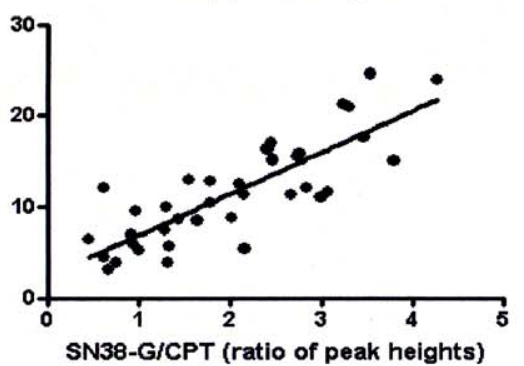

Figure 6. Association between glucuronidation of belinostat and UGT1A1 substrates (A, B, and C). A: Bilirubin-G; B: thyroxine-4-G/CPT11; C: SN38-G/CPT11.

doi:10.1371/journal.pone.0054522.g006

tion occurring at the hydroxamate moiety, leading to inactivation of its cytotoxicity. This is consistent among other hydroxamate class HDAC inhibitors as well, where glucuronidation plays a significant role in their metabolism. The metabolite profiling after incubation with a panel of supersomes overexpressing specific UGT isoforms, correlation with glucuronidation rates of known UGT1A1 substrates using human liver microsomes, and analysis of the mass spectrometry profile of belinostat in a cohort of patients with liver cancer provided evidence that belinostat glucuronidation in vitro and in vivo is mediated primarily by UGT1A1. Interestingly, the enzyme isoform that primarily metabolises another hydroxamic based HDAC inhibitor vorinostat in vivo is UGT2B17, whereas belinostat is metabolised by UGT1A1, which is a more abundant isoform in the human liver [31]. UGT1Al is present in the gastrointestinal tract, which would likely reduce oral absorption of belinostat, and lead to reduced bioavailability [32]. In addition, the high efficiency of UGT1A1 for metabolising belinostat will likely reduce further its oral bioavailability through extensive first pass metabolism, posing a challenge to development of oral belinostat.

The HDAC inhibitors that are in clinical development have potential for serious side effects. Some class related effects include thrombocytopenia, prolonged QT interval on the electrocardiogram, severe fatigue and gastrointestinal side effects, and these appear to be dose dependent. Belinostat has been associated with fatigue, diarrhea and prolongation of QT interval in phase I and II trials $[11,33]$. In view of its narrow therapeutic window, it is important to determine the factors such as pharmacogenetics of belinostat that account for interindividual variability of its pharmacokinetics. We previously showed that patients with deleted UGT2B17 alleles have lower vorinostat-glucuronide to vorinostat area-under-the-curve ratio and greater side effects [20]. Accordingly, it would be important to understand the extent of interindividual variability of belinostat pharmacokinetics and pharmacodynamics attributable to polymorphic expression of UGT1A1. Our study in human liver microsomes is the first step in this direction.

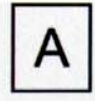

$$
\text { UGTIA1 MRNA }
$$$$
\left(r^{2}=0.66, p<0.0001, n=33\right)
$$

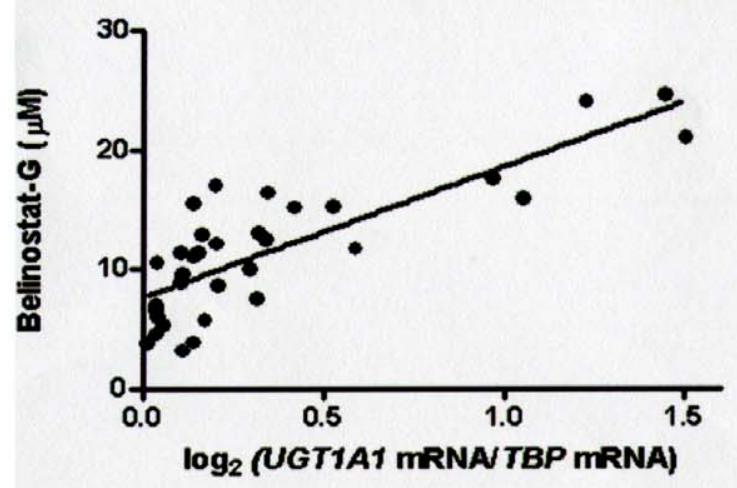

B

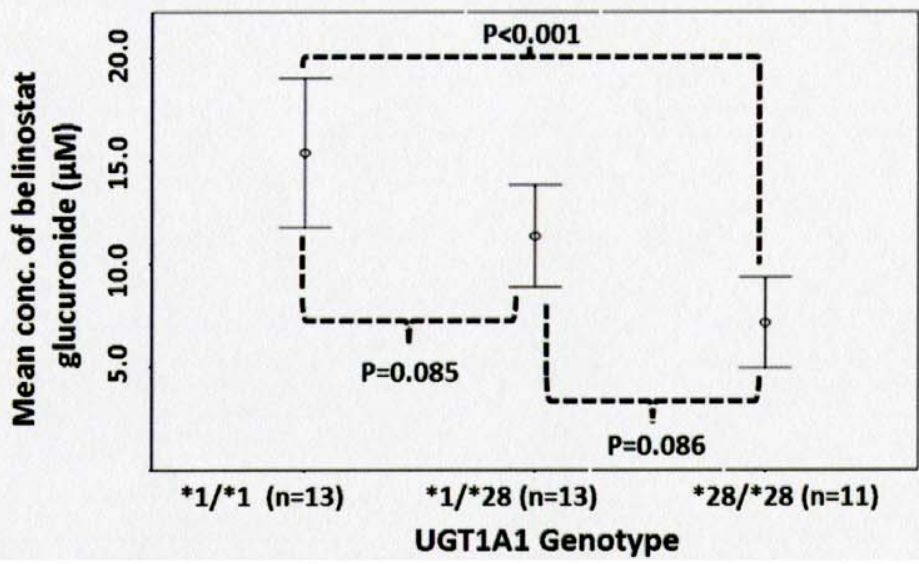

Figure 7. UGT1A1 expression on belinostat glucuronidation and impact of the common UGT1A1*28 promoter polymorphism. A: Correlation of belinostat glucuronide formation with UGT1A1 expression in human liver microsomes; B: Belinostat glucuronide formation by human liver microsomes according to wild-type, heterozygous and homozygous UGT1A $1 * 28$ genotypes. doi:10.1371/journal.pone.0054522.g007 
UGT1A1*28 is a genetic polymorphism at the promoter region where an additional TA repeat is present in the TATA box that usually has 6 TA repeats, and results in reduced expression of UGT1A1 [34]. In human liver microsomes, glucuronidation of $\mathrm{SN}-38$, the active metabolite of irinotecan, is less efficient in microsomes harbouring UGT1A1*28 compared to wildtype genotype [25]. Concordantly, individuals with one or more UGT1A $1 * 28$ alleles experience higher risk of neutropenia from irinotecan treatment at doses higher than $250 \mathrm{mg} / \mathrm{m}^{2}[35,36]$. This polymorphism is also more common in Caucasians than Asians, with an allelic frequency of about $30 \%$ in Caucasians and $10 \%$ in Asians [37]. Our data shows that UGT1A1*28 is associated with reduced belinostat glucuronidation, which suggests that patients with this genotype may potentially have higher exposure to active belinostat resulting from impaired clearance of belinostat. Though the clinical consequences of this higher exposure with the recommended dose of belinostat are currently unknown, higher toxicities to belinostat are expected. If this is proven, UGT1A1 genotyping prior to belinostat therapy, as is currently available for irinotecan, is a way to individualise therapy.

Whether UGT1A1*28 influences the pharmacokinetics and more crucially the pharmacodynamics of belinostat in patients remains to be studied; our data supports the need for such future studies in patients. In addition, the relevance of other polymorphisms of UGT1Al should be studied further, as there is substantial ethnogeographical variability of UGT1Al variants. For example, UGT1A1*28 is less common in East Asians, where UGT1A1*6 (Gly71R) is more common, and associated with reduced enzymatic activity.

\section{References}

1. Marks PA, Richon VM, Miller T, Kelly WK (2004) Histone deacetylase inhibitors. Adv Cancer Res 91: 137-168.

2. Gray SG, Qian CN, Furge K, Guo X, The BT (2004) Microarray profiling of the effects of histone deacetylase inhibitors on gene expression in cancer cell lines. Int J Oncol 24: 773-795.

3. Mitsiades CS, Mitsiades NS, Mcmullan CJ, Poulaki V, Shringarpure R, et al. (2004) Transcriptional signature of histone deacetylase inhibition in multiple myeloma: biological and clinical implications. Proc Natl Acad Sci 101: 540-545.

4. Peart MJ, Smyth GK, van Laar RK, Bowtell DD, Richon VM, et al. (2005) Identification and functional significance of genes regulated by structurally different histone deacetylase inhibitors. Proc Natl Acad Sci 102: 3697-3702.

5. Tanji N, Ozawa A, Kikugawa T, Miura N, Sasaki T, et al. (2011) Potential of histone deacetylase inhibitors for bladder cancer treatment. Expert Rev Anticancer Ther 1: 959-965.

6. Bapat SA (2010) Modulation of gene expression in ovarian cancer by active and repressive histone marks. Epigenomics 2: 39-51.

7. Piekarz RL, Bates SE (2009) Epigenetic modifiers: basic understanding and clinical development. Clin Cancer Res 15: 3918-3926.

8. Piekarz RL, Frye R, Prince HM, Kirschbaum MH, Zain J, et al. (2011) Phase 2 trial of romidepsin in patients with peripheral T-cell lymphoma. Blood. 117: 5827-5834.

9. Mann BS, Johnson JR, Cohen MH, Justice R, Pazdur R (2007) FDA Approval Summary: Vorinostat for Treatment of Advanced Primary Cutaneous T-Cell Lymphoma. The Oncologist. 12: 1247-1252.

10. Howman RA, Prince HM (2011) New drug therapies in peripheral T-cell lymphoma. Expert Rev Anticancer Ther 11: 457-472.

11. Steele NL, Plumb JA, Vidal L, Tjornelund J, Knoblauch P, et al. (2008) A Phase 1 Pharmacokinetic and Pharmacodynamic Study of the Histone Deacetylase Inhibitor Belinostat in Patients with Advanced Solid Tumors. Clin Cancer Res 14: 804-810.

12. Na YS, Jung KA, Kim SM, Hong YS, Ryu MH, et al. (2011) The histone deacetylase inhibitor PXD101 increases the efficacy of irinotecan in in vitro and in vivo colon cancer models. Cancer Chemother Pharmacol 68: 389-398.

13. Ramalingam SS, Belani CP, Ruel C, Frankel P, Gitlitz B, et al. (2009) Phase II study of belinostat (PXD101), a histone deacetylase inhibitor, for second line therapy of advanced malignant pleural mesothelioma. J Thorac Oncol 4: 97101.

14. Tumber A, Collins LS, Petersen KD, Thougaard A, Dejligbjerg M, et al. (2007) The histone deacetylase inhibitor PXD101 synergises with 5-fluorouracil to inhibit colon cancer cell growth in vitro and in vivo. Cancer Chemother Pharmacol. 60: 275-283.
Given the significance of UGT1A1 in the metabolism of belinostat, it would be important to study the potential interactions of known UGT1Al inhibitors and inducers on belinostat PK and pharmacodynamics. This is especially pertinent when belinostat is given in combination with irinotecan, a cytotoxic drug which also undergoes glucuronidation by UGT1A1 [12]. In colorectal cancer cell lines, the combination of belinostat and irinotecan was highly synergistic, and it is conceivable that such a combination is planned in patients with colorectal cancers in the near future.

\section{Supporting Information}

\section{Protocol S1 Trial protocol. \\ (PDF)}

\section{Acknowledgments}

We would like to express our thanks to Mrs. Khoo Yok Moi (Department of Pharmacology, National University of Singapore, Singapore) and Ms. Eileen Tan (Department of Hematology \& Oncology, National University Health System, Singapore) for their kind help on this project.

\section{Author Contributions}

Conceived and designed the experiments: BCG LZW WY AC SA. Performed the experiments: LZW JR MYMC WLT JYAL SCW ALAW YKZ RL. Analyzed the data: BCG MJR LZW JR WY SCL PCH HSL AC ALAW YKZ RL. Contributed reagents/materials/analysis tools: WY MJR HSL PCH AC SA. Wrote the paper: BCG LZW MJR JR PCH HSL.

15. Dai Y, Chen S, Wang L, Pei XY, Kramer LB, et al. (2011) Bortezomib interacts synergistically with belinostat in human acute myeloid leukaemia and acute lymphoblastic leukaemia cells in association with perturbation in NF- $\mathrm{KB}$ and Bim. Br J Haematol. 152(2): 222-235.

16. Giaccone G, Rajan A, Berman A, Kelly RJ, Szabo E, et al. (2011) Phase II study of belinostat in patients with recurrent or refractory advanced thymic epithelial tumors. J Clin Oncol. 29: 2052-2059.

17. Cashen A, Juckett M, Jumonville A, Litzow M, Flynn PJ, et al. (2012) Phase II study of the histone deacetylase inhibitor belinostat (PXD101) for the treatment of myelodysplastic syndrome (MDS). Ann Hematol 91(1): 33-38.

18. Mackay HJ, Hirte H, Colgan T, Covens A, MacAlpine K, et al. (2010) Phase II trial of the histone deacetylase inhibitor belinostat in women with platinum resistant epithelial ovarian cancer and micropapillary (LMP) ovarian tumours. Eur J Cancer 46: 1573-1579.

19. Kang SP, Ramirez J, House L, Zhang W, Mirkov S, et al. (2010) A pharmacogenetic study of vorinostat glucuronidation. Pharmacogenet. Genomics 20: 638-641.

20. Wong NS, Seah EZ, Wang LZ, Yeo WL, Yap HL, et al. (2011) Impact of UDPgluconoryltransferase 2B17 genotype on vorinostat metabolism and clinical outcomes in Asian women with breast cancer. Pharmacogenet. Genomics 21(11): 760-768

21. Clive S, Woo MM, Stewart M, Nydam T, Hirawat S, et al. (2009) Elucidation of the metabolic and elimination pathways of panobinostat (LBH589) using $\left[{ }^{14} \mathrm{C}\right]-$ panobinostat. J Clin Oncol 27: 15s.

22. Shiraga T, Tozuka Z, Ishimura R, Kawamura A, Kagayama A (2005) Identification of cytochrome P450 enzymes involved in the metabolism of FK228, a potent histone deacetylase inhibitor, in human liver microsomes. Biol Pharm Bull. 28: 124-129.

23. Balliet RM, Chen G, Gallagher CJ, Dellinger RW, Sun D, et al. (2009) Characterization of UGTs active against SAHA and association between SAHA glucuronidation activity phenotype with UGT genotype. Cancer Res. 69: 29812989 .

24. Wang LZ, Chan D, Yeo W, Wan SC, Chan S, et al. (2010) A sensitive and specific liquid chromatography-tandem mass spectrometric method for determination of belinostat in plasma from liver cancer patients. J Chromatogr B Biomed Sci Appl. 878: 2409-2414.

25. Iyer L, Hall D, Das S, Mortell MA, Ramirez J, et al. (1999) Phenotype-genotype correlation of in vitro $\mathrm{SN}-38$ (active metabolite of irinotecan) and bilirubin glucuronidation in human liver tissue with UGT1A1 promoter polymorphism. Clin Pharmacol Ther 65: 576-582. 
26. Yoder Graber AL, Ramirez J, Innocenti F, Ratain MJ (2007) UGT1A1*28 genotype affects the in-vitro glucuronidation of thyroxine in human livers. Pharmacogenet Genomics 17: 619-627.

27. Innocenti F, Liu W, Chen P, Desai AA, Das S, et al. (2005) Haplotypes of variants in the UDP-glucuronosyltransferase $1 \mathrm{~A} 9$ and $1 \mathrm{~A} 1$ genes. Pharmacogenet Genomics 15: 295-301.

28. Babu SR, Lakshmi VM, Hsu FF, Zenser TV, Davis BB, et al. (1995) Glucuronidation of N-hydroxy metabolits of $\mathrm{N}$-acetylbenzidine. Carcinogenesis. 16: 3069-3074.

29. Marco C, Vijaya ML, Nikhil B, Davis BB, Owens IS, et al. (1999) Glucuronidation of benzidine and its metabolites by cDNA-expressed human UDP-glucuronosyltransferases and $\mathrm{pH}$ stability of glucuronides. Carcinogenesis. 20: 1963-1969.

30. Du LH, Musson DG, Wang AQ. (2005) High turbulence liquid chromatography online extraction and tandem mass spectrometry for the simultaneous determination of suberoylanilide hydroxamic acid and its two metabolites in human serum. Rapid Commun Mass Spectrom 19: 1779-1787.

31. Izukawa T, Nakajima M, Fujiwara R, Yamanaka H, Fukami T, et al. (2009) Quantitative analysis of UDP-glucuronosyltransferase (UGT) 1A and UGT2B expression levels in human livers. Drug Metab Dispos. 37: 1759-1768.
32. Strassburg CP, Manns MP, Tukey RH (1998) Expression of the UDPglucuronosyltransferase $1 \mathrm{~A}$ locus in human colon. Identification and characterization of the novel extrahepatic UGT1A8. J Biol Chem. 273: 8719-8726.

33. Yeo W, Chung HC, Chan SL, Wang LZ, Lim R, et al. (2012) Epigenetic Therapy Using Belinostat for Patients With Unresectable Hepatocellular Carcinoma: A Multicenter Phase I/II Study With Biomarker and Pharmacokinetic Analysis of Tumors From Patients in the Mayo Phase II Consortium and the Cancer Therapeutics Research Group. J Clin Oncol. 30: 3361-3367.

34. Bosma PJ, Chowdhury JR, Bakker C, Gantla S, de Boer A, et al. (1995) The genetic basis of the reduced expression of bilirubin UDP-glucuronosyltransferase 1 in Gilbert's syndrome. N Engl J Med 333: 1171-1175.

35. Innocenti F, Undevia SD, Iyer L, Chen PX, Das S, et al. (2004) Genetic variants in the UDP-glucuronosyltransferase $1 \mathrm{Al}$ gene predict the risk of severe neutropenia of irinotecan. J Clin Oncol. 22: 1382-1388.

36. Hoskins JM, Goldberg RM, Qu P, Ibrahim JG, McLeod HL (2007) UGT1A1*28 genotype and irinotecan-induced neutropenia: dose matters. J Natl Cancer Inst. 99: 1290-1295.

37. Beutler E, Gelbart T, Demina A (1998) Racial variability in the UDPglucuronosyltransferase 1 (UGT1A1) promoter: a balanced polymorphism for regulation of bilirubin metabolism? Proc Natl Acad Sci U S A. 95: 8170-8174. 